\title{
IMPEDANCE PASSIVE AND CONSERVATIVE BOUNDARY CONTROL SYSTEMS
}

\author{
JARMO MALINEN AND OLOF J. STAFFANS
}

\begin{abstract}
The external Cayley transform is used for the conversion between the linear dynamical systems in scattering form and in impedance form. We use this transform to define a class of formal impedance conservative boundary control systems (colligations), without assuming a priori that the associated Cauchy problems are solvable. We give sufficient and necessary conditions when impedance conservative colligations are internally well-posed boundary nodes; i.e. when the associated Cauchy problems are solvable and governed by $C_{0}$ semigroups. We define a "strong" variant of such colligations, and we show that "strong" impedance conservative boundary colligation is a slight generalization of the "abstract boundary space" construction for a symmetric operator in the Russian literature. Many aspects of the theory is illustated by examples involving the transmission line and the wave equations.
\end{abstract}

\section{INTRODUCTION}

This paper deals with linear boundary control/observation systems described by differential equations of the form

$$
\begin{aligned}
& u(t)=G z(t), \\
& \dot{z}(t)=L z(t), \\
& y(t)=K z(t), \quad t \in \mathbb{R}^{+}=[0, \infty), \\
& z(0)=z_{0} .
\end{aligned}
$$

In a typical application $L$ is a partial differential operator on a bounded domain $\Omega \subset \mathbb{R}^{n}$, and $G$ and $K$ are composed of some boundary trace operators on $\partial \Omega$.

Let us explain the purpose of this paper with a concrete example. Suppose we wish to compute the total impedance of an electical transmission line, described by the coupled first order PDEs

$$
\left\{\begin{array}{c}
\frac{\partial}{\partial t}\left[\begin{array}{c}
I(\xi, t) \\
U(\xi, t)
\end{array}\right]=\left[\begin{array}{cc}
0 & -\frac{1}{L(\xi)} \frac{\partial}{\partial \xi} \\
-\frac{1}{C(\xi)} \frac{\partial}{\partial \xi} & 0
\end{array}\right]\left[\begin{array}{l}
I(\xi, t) \\
U(\xi, t)
\end{array}\right] \\
\text { for }(\xi, t) \in[0,1] \times \overline{\mathbb{R}}_{+} \\
u(t)=I(0, t) \text { for } t \in \overline{\mathbb{R}}_{+} \text {and } y(t)=U(0, t) \text { for } t \in \overline{\mathbb{R}}_{+} \\
I(\xi, 0)=U(\xi, 0)=0 \text { for } \xi \in[0,1] \text { and } I(1, t)=0 \text { for } t \in \overline{\mathbb{R}}_{+}
\end{array}\right.
$$

These equations are clearly of the form (1.1). The real-valued, continuously differentiable functions $L(\xi)$ and $C(\xi)$ are the distributed inductances and the capacitances of the line at point $\xi$, and it will be assumed that $L(\xi) \geq \eta$ and $C(\xi) \geq \eta$ for some $\eta>0$. The functions $I(\xi, t)$ and $U(\xi, t)$ are the current and the voltage at point $\xi$ at time $t$, respectively. It is thus clear that the transfer function of (1.2) represents 
the total impedance $\hat{Z}(s)$ of the line, provided we can make sense out of (1.2) as an infinite-dimensional state space system with input $u(\cdot)$ and output $y(\cdot)$.

The setting described above can be technically difficult to deal with. By choosing the signals in a different way we get a system with better mathematical properties. Instead of (1.2) we can study the scattering system described by

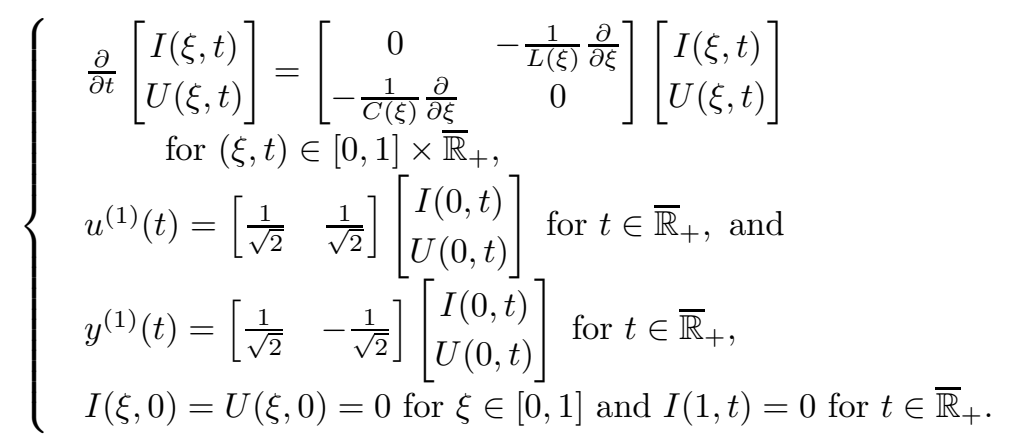

Also these equations are of the form (1.1). Since equations (1.3) do not include energy dissipative resistances, it is physically plausible (and we prove it in Section 6) that they define a scattering conservative, well-posed boundary control system with input $u^{(1)}(\cdot)$ and output $y^{(1)}(\cdot)$. Such systems are described in [Mal05, MS06a]; see also [MSW06].

In system theory, the translation from (1.2) to (1.3) is known as the external Cayley transform. Also the name diagonal transform has been used in the Russian literature; see [Liv73]. The external Cayley transform can be applied to a much larger class of linear infinite-dimensional systems than just the transmission line described above; see Definition 3.1 below.

Suppose that the triple $\Xi^{(1)}=\left[\begin{array}{c}G^{(1)} \\ L \\ K^{(1)}\end{array}\right]$ is the external Cayley transform of the triple $\Xi=\left[\begin{array}{c}G \\ L \\ K\end{array}\right]$. Even when $\Xi^{(1)}$ is known to be a scattering conservative boundary node, two difficulties may appear:

- There is no guarantee that the triple $\Xi$ itself is a (forward time) boundary node in the sense of Definition 2.2. (If it is, then it is internally well-posed in the sense that it has a strongly continuous semigroup; see Proposition 4.1.)

- Even if such a triple $\Xi$ were an internally well-posed boundary node, it need not define a well-posed linear system.

The main purpose of this paper is to treat the first of these problems in an abstract framework that includes boundary control systems. We discuss connections to the earlier results on the abstract boundary spaces as defined [GG91] and the references therein. Finally, in Section 6 we return to the example described above.

\section{Scattering Passive Nodes}

In this section we study the scattering case. We define the notion of a scattering passive or conservative boundary node, and then we give a necessary and sufficient conditions for a triple of operators $G, L$, and $K$ to form a node of one of these types. We then move on to reformulate these conditions in terms of geometric conditions in a particular Kreĭn space. These conditions will be important for our treatment of the impedance case in the next section. 
We begin by introducing some terminology and defining a boundary node in general.

Definition 2.1. A colligation $\Xi:=\left(\left[\begin{array}{l}G \\ L \\ K\end{array}\right] ;\left[\begin{array}{c}\mathcal{U} \\ \mathcal{X} \\ \mathcal{Y}\end{array}\right]\right)$ consists of the three Hilbert spaces $\mathcal{U}, \mathcal{X}$, and $\mathcal{Y}$, and the three linear maps $G, L$, and $K$, with the same domain $\mathcal{Z} \subset \mathcal{X}$ and with values in $\mathcal{U}, \mathcal{X}$, and $\mathcal{Y}$, respectively. By the domain $\mathcal{D}$ om $(\Xi)$ of $\Xi$ we mean the common domain $\mathcal{Z}$ of $G, L$, and $K$. This colligation is closed if $\left[\begin{array}{l}G \\ L \\ K\end{array}\right]$ is closed as an operator $\mathcal{X} \rightarrow\left[\begin{array}{l}\mathcal{U} \\ \mathcal{X} \\ \mathcal{Y}\end{array}\right]$ with domain $\mathcal{Z}$.

Definition 2.2. A colligation $\Xi:=\left(\left[\begin{array}{c}G \\ L \\ K\end{array}\right] ;\left[\begin{array}{c}\mathcal{U} \\ \mathcal{X} \\ \mathcal{Y}\end{array}\right]\right)$ is a boundary node if the following conditions are satisfied:

(i) $\Xi$ is closed;

(ii) $G$ is surjective and $\mathcal{N}(G)$ is dense in $\mathcal{X}$;

(iii) The operator $A:=\left.L\right|_{\mathcal{N}(G)}$ has a nonempty resolvent set.

This boundary node is internally well-posed (in the forward time direction) if, in addition,

(iv) A generates a $C_{0}$ semigroup.

We call $\mathcal{U}$ the input space, $\mathcal{X}$ the state space, $\mathcal{Y}$ the output space, $\mathcal{Z}$ the solution space, $G$ the input boundary operator, $L$ the interior operator, $K$ the output boundary operator, and $A$ the main operator (or the semigroup generator in the internally well-posed case).

If $\Xi=\left(\left[\begin{array}{c}G \\ L \\ K\end{array}\right] ;\left[\begin{array}{l}\mathcal{U} \\ \mathcal{X}\end{array}\right]\right)$ is an internally well-posed boundary node, then (1.1) has a unique solution for sufficiently smooth input functions $u$ and initial states $z_{0}$ compatible with $u(0)$. More precisely, as shown in [MS06a, Lemma 2.6], for all $z_{0} \in \mathcal{X}$ and $u \in C^{2}\left(\mathbb{R}^{+} ; \mathcal{U}\right)$ with $G z_{0}=u(0)$ the first, second and fourth of the equations (1.1) have a unique solution $z \in C^{1}\left(\mathbb{R}^{+} ; \mathcal{X}\right) \cap C\left(\mathbb{R}^{+} ; \mathcal{Z}\right),{ }^{1}$ and hence we can define $y \in C\left(\mathbb{R}^{+} ; \mathcal{Y}\right)$ by the third equation in (1.1). In the rest of this article, when we say "a smooth solution of $(1.1)$ on $\mathbb{R}^{+}$" we mean a solution with the above properties.

Definition 2.3. A boundary node $\Xi$ on $(\mathcal{U}, \mathcal{X}, \mathcal{Y})$ is scattering passive if it is internally well-posed and all smooth solutions of $(1.1)$ on $\mathbb{R}^{+}$satisfy

$$
\frac{\mathrm{d}}{\mathrm{d} t}\|z(t)\|_{\mathcal{X}}^{2}+\|y(t)\|_{\mathcal{Y}}^{2} \leq\|u(t)\|_{\mathcal{U}}^{2}, \quad t \in \mathbb{R}^{+}
$$

It is scattering energy-preserving if the above inequality holds in the form of an equality.

Following [MS06a], we define scattering conservativity by means of time-flow inversion.

Definition 2.4. A boundary node $\Xi=\left(\left[\begin{array}{c}G \\ L \\ K\end{array}\right] ;\left[\begin{array}{c}\mathcal{U} \\ \mathcal{X}\end{array}\right]\right)$ is time-flow invertible if the colligation $\Xi^{\leftarrow}:=\left(\left[\begin{array}{c}K \\ -L \\ G\end{array}\right] ;\left[\begin{array}{c}\mathcal{Y} \\ \mathcal{X} \\ \mathcal{U}\end{array}\right]\right)$ is a boundary node. The node $\Xi$ is scattering conservative if it is time-flow invertible and both $\Xi$ itself and the time-flow inverse $\Xi^{\leftarrow}$ are energy preserving.

\footnotetext{
${ }^{1}$ Here we use the graph norm of $\left[\begin{array}{c}G \\ L \\ K\end{array}\right]$ in $\operatorname{Dom}(\Xi)$.
} 
The following theorem gives necessary and sufficient conditions for a colligation $\Xi$ to be a scattering passive boundary node.

Theorem 2.5. A colligation $\Xi=\left(\left[\begin{array}{l}G \\ L \\ K\end{array}\right] ;\left[\begin{array}{l}\mathcal{U} \\ \mathcal{X} \\ \mathcal{Y}\end{array}\right]\right)$ is a scattering passive boundary node if and only if it satisfies the following two conditions:

(i) $\left[\begin{array}{c}G \\ \alpha-L\end{array}\right]$ is surjective for some $\alpha \in \mathbb{C}^{+}:=\{z \in \mathbb{C} \mid \operatorname{Re} z>0\}$;

(ii) For all $z \in \mathcal{D}$ om $(\Xi)$ we have

$$
2 \operatorname{Re}\langle z, L z\rangle_{\mathcal{X}}+\|K z\|_{\mathcal{Y}}^{2} \leq\|G z\|_{\mathcal{U}}^{2}
$$

The colligation $\Xi$ is a scattering energy preserving boundary node if and only if it is scattering passive and (2.2) holds in the form of an equality, i.e,

$$
2 \operatorname{Re}\langle z, L z\rangle_{\mathcal{X}}+\|K z\|_{\mathcal{Y}}^{2}=\|G z\|_{\mathcal{U}}^{2}, \quad z \in \operatorname{Dom}(\Xi)
$$

Finally, $\Xi$ is a scattering conservative boundary node if and only if it is an energy preserving boundary node and, in addition,

(iii) $\left[\begin{array}{c}\gamma-L \\ K\end{array}\right]$ is surjective for some $\gamma \in \mathbb{C}^{-}:=\{z \in \mathbb{C} \mid \operatorname{Re} z<0\}$.

If $\Xi$ is scattering passive, then the semigroup generated by $\left.L\right|_{\mathcal{N}(G)}$ is a contraction semigroup and condition (i) holds for all $\alpha \in \mathbb{C}^{+}$. If $\Xi$ is scattering conservative, then the semigroup generated by $-\left.L\right|_{\mathcal{N}(K)}$ is a contraction semigroup and condition (iii) holds for all $\gamma \in \mathbb{C}^{-}$.

Clearly, condition (i) in Theorem 2.5 holds if and only if both $G$ and $\alpha-A$ are surjective, where $A:=\left.L\right|_{\mathcal{N}(G)}$.

Proof. Suppose first that $\Xi$ is a scattering passive boundary node. Then condition (ii) in Definition 2.2 says that $G$ is surjective, and condition (iv) in Definition 2.2 implies that $(\alpha-A)$ is surjective for all $\alpha$ with $\operatorname{Re} \alpha$ large enough. Thus, condition (i) in Theorem 2.5 holds. To verify condition (ii) in Theorem 2.5 we take any $z_{0} \in \operatorname{Dom}(\Xi)$ and let $(u, z, y)$ be the smooth solution of (1.1) with initial state $z(0)=z_{0}$ and constant input function $u(t)=G z_{0}$ for $t \geq 0$ (see [MS06a, Lemma 2.6]). Then (1.1) and (2.1) with $t=0$ imply (2.2) with $z$ replaced by $z_{0}$ (since $\left.\frac{\mathrm{d}}{\mathrm{d} t}\|z(t)\|_{\mathcal{X}}^{2}=2 \operatorname{Re}\langle z(t), \dot{z}(t)\rangle_{\mathcal{X}}\right)$. Thus, (2.2) holds for all $z \in \mathcal{D}$ om $(\Xi)$.

Conversely, suppose that the assumption of Theorem 2.5 holds. We claim that $A$ is maximal dissipative, and hence it generates a $C_{0}$ contraction semigroup (in particular, $\mathcal{N}(G)$ is dense in $\mathcal{X})$. Without loss of generality, we may assume that $\alpha$ in condition (i) is real and positive (otherwise we replace $\alpha$ by $\operatorname{Re} \alpha$ and $A$ by $A-i \operatorname{Im} \alpha$ ). It then follows from [Paz83, Theorems 1.4.3 and 1.4.6] that $A$ is maximal dissipative, and that it generates a constraction semigroup. This implies that $\mathbb{C}^{+} \in \rho(A)$ (see, e.g., [Sta05, 3.2.9]), and hence (i) holds for all $\alpha \in \mathbb{C}^{+}$.

That $\Xi$ is closed will be shown as a part of the proof of Lemma 2.6. Taking this for granted, we find that the assumption of Theorem 2.5 imples that $\Xi$ is an internally well-posed boundary node. That also (2.1) holds follows from (1.1) and (2.2), and we conclude that $\Xi$ is a scattering passive boundary node.

The proofs for the energy-preserving case and conservative case are analogous, and they are left to the reader.

In the next two lemmas, we characterize a scattering passive, energy preserving, or conservative boundary node $\Xi$ in terms of the Kre ̌n space structure of the graph of $\Xi$. For that reason, let us first recall some definitions and facts about Kre ̌n spaces. 
Let $\mathfrak{H}$ be a Hilbert space with the inner product $\langle\cdot, \cdot\rangle$, and let $J=J^{*}=J^{-1}$ be a signature operator in $\mathfrak{H}$. Denote by $\mathfrak{K}$ the resulting Kre $\breve{n}$ space that we get from $\mathfrak{H}$ by using the (possibly) nondefinite inner product $[\cdot, \cdot]=\langle\cdot, J \cdot\rangle$. The nonpositivity of a subspace $V \subset \mathfrak{K}$ means that $[z, z] \leq 0$ for all $z \in V$. Such a $V$ is maximal nonpositive if it is not included in a strictly larger nonpositive subspace of $\mathfrak{K}$. A maximal nonpositive subspace is always closed. Nonnegative and maximal nonnegative subspaces are defined analogously. A subspace $V \subset \mathfrak{K}$ is called neutral if $[z, z]=0$ for all $z \in V$. Writing $V^{[\perp]}:=\{z \in \mathfrak{K}:[z, v]=0$ for all $v \in V\}$ for the Kreln orthogonal companion, we see (by the polarization identity) that $V$ is neutral if and only if $V \subset V^{[\perp]}$. If $V=V^{[\perp]}$, then $V$ is called Lagrangian. A subspace is Lagrangean if and only if it is both maximal nonnegative and maximal nonpositive (see [Bog74, Theorem 7.4, p. 15]).

Let now $\mathfrak{K}^{\prime}$ be another Krein space with inner product $[\cdot, \cdot]^{\prime}$. We say that a bounded operator $U: \mathfrak{K} \rightarrow \mathfrak{K}^{\prime}$ is (Krĕ̌n) unitary if it is a bijection and $\left[U z_{1}, U z_{2}\right]^{\prime}=$ $\left[z_{1}, z_{2}\right]$ for all $z_{1}, z_{2} \in \mathfrak{K}$. We shall use several times the following easily verified facts: Such $U$ maps (maximal) nonpositive subspaces onto (maximal) nonpositive subspaces, (maximal) nonnegative subspaces onto (maximal) nonnegative subspaces, neutral subspaces onto neutral subspaces, and Lagrangian subspaces onto Lagrangian subspaces.

By the graph $\mathcal{G}(\Xi)$ of a colligation $\Xi=\left(\left[\begin{array}{l}G \\ L \\ K\end{array}\right] ;\left[\begin{array}{l}\mathcal{U} \\ \mathcal{Y}\end{array}\right]\right)$ we mean the set

$$
\mathcal{G}(\Xi)=\left\{\left[\begin{array}{l}
u \\
z \\
v \\
y
\end{array}\right] \in\left[\begin{array}{l}
\mathcal{U} \\
\mathcal{X} \\
\mathcal{Y}
\end{array}\right] \mid\left[\begin{array}{l}
u \\
v \\
y
\end{array}\right]=\left[\begin{array}{l}
G \\
L \\
K
\end{array}\right] z, z \in \operatorname{Dom}(\Xi)\right\} .
$$

Clearly, this graph is closed in $\left[\begin{array}{l}\mathcal{U} \\ \mathcal{X} \\ \mathcal{Y}\end{array}\right]$ if and only if $\Xi$ is closed.

Lemma 2.6. The colligation $\Xi=\left(\left[\begin{array}{l}G \\ L \\ K\end{array}\right] ;\left[\begin{array}{l}\mathcal{U} \\ \mathcal{Y}\end{array}\right]\right)$ is a scattering passive boundary node if and only if $\mathcal{G}(\Xi)$ is a maximal nonpositive subspace of the Kre ̌n space $\mathfrak{K}_{s c}$ induced by the signature operator $J_{s c}:=\left[\begin{array}{cccc}-1 & 0 & 0 & 0 \\ 0 & 0 & 1 & 0 \\ 0 & 1 & 0 & 0 \\ 0 & 0 & 0 & 1\end{array}\right]$ in the Hilbert space $\left[\begin{array}{l}\mathcal{U} \\ \mathcal{X} \\ \mathcal{Y}\end{array}\right]$.

Proof. We begin by showing that $\mathcal{G}(\Xi)$ is maximal nonpositive if and only if the assumption of Theorem 2.5 holds. It is easy to see that condition (ii) in Theorem 2.5 holds if and only if $\mathcal{G}(\Xi)$ is nonpositive in $\mathfrak{K}_{s c}$. In the sequel we assume these equivalent conditions to hold, and show that then $\mathcal{G}(\Xi)$ is maximal nonpositive if and only if condition (i) in Theorem 2.5 holds.

It is easy to see that for each $\alpha \in \mathbb{C}^{+}$, the operator

$$
U_{\alpha}^{i n t}:=\left[\begin{array}{cccc}
1 & 0 & 0 & 0 \\
0 & \frac{1}{\sqrt{2 \operatorname{Re} \alpha}} & 0 & 0 \\
0 & 0 & \frac{1}{\sqrt{2 \operatorname{Re} \alpha}} & 0 \\
0 & 0 & 0 & 1
\end{array}\right]\left[\begin{array}{cccc}
1 & 0 & 0 & 0 \\
0 & \alpha & -1 & 0 \\
0 & \alpha & 1 & 0 \\
0 & 0 & 0 & 1
\end{array}\right]
$$

is a (Krĕn) unitary operator from $\mathfrak{K}_{s c}$ to the Kreın space $\mathfrak{K}_{s c}^{i}$ induced by the signature operator $J_{s c}^{i}:=\left[\begin{array}{cccc}-1 & 0 & 0 & 0 \\ 0 & -1 & 0 & 0 \\ 0 & 0 & 1 & 0 \\ 0 & 0 & 0 & 1\end{array}\right]$ in $\left[\begin{array}{l}\mathcal{U} \\ \mathcal{X} \\ \mathcal{Y}\end{array}\right]$. Thus, $\mathcal{G}(\Xi)$ is maximal nonpositive in $\mathfrak{K}_{s c}$ if and only if the image

$$
U_{\alpha}^{i n t} \mathcal{G}(\Xi)=\left\{\left[\begin{array}{c}
1 / \sqrt{2 \operatorname{Re} \alpha}(\alpha-L) z \\
1 / \sqrt{2 \operatorname{Re} \alpha}(\bar{\alpha}+L) z \\
K z
\end{array}\right] \mid z \in \operatorname{Dom}(\Xi)\right\}
$$


is maximal nonpositive in $\mathfrak{K}_{s c}^{i}$.

Because of condition (ii) in Theorem 2.5, the operator $[1 / \sqrt{2 \operatorname{Re} \alpha}(\alpha-L)]$ is injective. Therefore the image $U_{\alpha}^{i n t} \mathcal{G}(\Xi)$ is the graph of an operator $T$ from $\operatorname{Dom}(T)=$ $\mathcal{R}\left(\left[\begin{array}{l}G / \sqrt{2 \operatorname{Re} \alpha}(\alpha-L)\end{array}\right]\right)$ into $\left[\begin{array}{l}\mathcal{X} \\ \mathcal{Y}\end{array}\right]$, and this operator is a contraction since $U_{\alpha}^{i n t} \mathcal{G}(\Xi)$ is nonpositive in $\mathfrak{K}_{s c}^{i}$. According to [Bog74, Theorem 11.7, p. 54 and Theorem 4.2, p. 105], $U_{\alpha}^{\text {int }} \mathcal{G}(\Xi)$ is maximally nonnegative if and only if $\operatorname{Dom}(T)=\left[\begin{array}{l}\mathcal{U} \\ \mathcal{X}\end{array}\right]$, and this is true if and only if condition (i) in Theorem 2.5 holds.

We have now shown that $\mathcal{G}(\Xi)$ is maximal nonnegative if and only if the assumption of Theorem 2.5 holds. Since every maximal nonnegative subspace is closed (see [Bog74, Theorem 4.1, p. 105]), this means that the assumption of Theorem 2.5 implies that $\Xi$ is closed, and we have thereby completed the proof of Theorem 2.5. The conclusion of the present lemma follows immediately.

Lemma 2.7. Let $\Xi=\left(\left[\begin{array}{l}G \\ L \\ K\end{array}\right] ;\left[\begin{array}{l}\mathcal{U} \\ \mathcal{X} \\ \mathcal{Y}\end{array}\right]\right)$ be a colligation.

(i) $\Xi$ is a scattering energy preserving boundary node if and only if $\mathcal{G}(\Xi)$ is a neutral and maximal nonpositive subspace of the Krein space $\mathfrak{K}_{s c}$ defined in Lemma 2.6.

(ii) $\Xi$ is a scattering conservative boundary node if and only if $\mathcal{G}(\Xi)$ is a maximal nonpositive and maximal nonnegative subspace of the Krein space $\mathfrak{K}_{s c}$ defined in part (iii) of Lemma 2.6, or equivalently, if and only if $\mathcal{G}(\Xi)$ is a Lagrangian subspace of $\mathfrak{K}_{s c}$.

The proof of this proposition is analogous to the proof of Lemma 2.6, and we leave it to the reader.

\section{Impedance Passive Nodes with $\mathcal{U}=\mathcal{Y}$}

As we mentioned in the introduction, we shall study impedance type systems by transforming them into scattering type systems by means of the external Cayley transform. We assume first that the output space $\mathcal{Y}$ is equal to the input space $\mathcal{U}$.

Definition 3.1. By the external Cayley transform of $\Xi$ with parameter $\beta \in \mathbb{C}^{+}$of the colligation $\Xi=\left(\left[\begin{array}{l}G \\ L \\ K\end{array}\right] ;\left[\begin{array}{l}\mathcal{U} \\ \mathcal{U}\end{array}\right]\right)$ we mean the colligation $\Xi^{(\beta)}=\left(\left[\begin{array}{c}G^{(\beta)} \\ L \\ K^{(\beta)}\end{array}\right] ;\left[\begin{array}{c}\mathcal{U} \\ \mathcal{X}\end{array}\right]\right)$ with $\operatorname{Dom}\left(\Xi^{(\beta)}\right)=\operatorname{Dom}(\Xi)$, where

$$
G^{(\beta)}=\frac{\beta G+K}{\sqrt{2 \operatorname{Re} \beta}} \quad \text { and } \quad K^{(\beta)}=\frac{\bar{\beta} G-K}{\sqrt{2 \operatorname{Re} \beta}} .
$$

The interpretation of this transform is the following: the old input $u$ and the old output $y$ in (1.1) are replaced by the new input $u^{(\beta)}=(\beta u+y) / \sqrt{2 \operatorname{Re} \beta}$ and the new output $y^{(\beta)}=(\bar{\beta} u-y) / \sqrt{2 \operatorname{Re} \beta}$. In the case of the transmission line example in Section 1, the external Cayley transform (with $\beta=1$ ) takes the form of Figure 1 below where $\hat{Z}(s)$ for $s \in \mathbb{C}^{+}$denotes the total impedance of the transmission line. ${ }^{2}$

We define the class of impedance passive colligations by reducing it to the scattering case by means of the external Cayley transform:

\footnotetext{
${ }^{2}$ This $\hat{Z}(s)$ equals the transfer function of the system node defined by $(1.2)$.
} 
Definition 3.2. Let $\Xi=\left(\left[\begin{array}{c}G \\ L \\ K\end{array}\right] ;\left[\begin{array}{l}\mathcal{U} \\ \mathcal{X} \\ \mathcal{U}\end{array}\right]\right)$ be a colligation, and let $\Xi^{(\beta)}=\left(\left[\begin{array}{c}G^{(\beta)} \\ L \\ K^{(\beta)}\end{array}\right] ;\left[\begin{array}{l}\mathcal{U} \\ \mathcal{X}\end{array}\right]\right)$ be the external Cayley transform of $\Xi$ with parameter $\beta$.

(i) $\Xi$ is impedance passive if $\Xi^{(\beta)}$ is a scattering passive boundary node for some $\beta \in \mathbb{C}^{+}$.

(ii) $\Xi$ is impedance energy preserving if $\Xi^{(\beta)}$ is a scattering energy preserving boundary node for some $\beta \in \mathbb{C}^{+}$.

(iii) $\Xi$ is impedance conservative if $\Xi^{(\beta)}$ is a scattering conservative boundary node for some $\beta \in \mathbb{C}^{+}$.

We remark that the operators $G$ and $K$ of an impedance passive colligation $\Xi=\left(\left[\begin{array}{l}G \\ L \\ K\end{array}\right] ;\left[\begin{array}{l}\mathcal{U} \\ \mathcal{X}\end{array}\right]\right)$ satisfy $G, K \in \mathcal{L}(\mathcal{Z} ; \mathcal{U})$ when $\mathcal{Z}=\mathcal{D}$ om $(\Xi)$ is equipped with the graph norm of $\left[\begin{array}{c}G \\ L \\ K\end{array}\right]$. This holds because the graph norms of $\left[\begin{array}{c}G \\ L \\ K\end{array}\right]$ and $\left[\begin{array}{c}G^{(\beta)} \\ L \\ K^{(\beta)}\end{array}\right]$ are equivalent on $\mathcal{Z}$ for all $\beta \in \mathbb{C}^{+}$.

As will be shown in Theorem 3.3 below, Definition 3.2 is independent of the parameter $\beta$ in the sense that if the conditions (i)-(iii) are true for some $\beta \in \mathbb{C}^{+}$, then they are true for all $\beta \in \mathbb{C}^{+}$. For the transmission line system of Section 1 , the parameter $\beta$ is the resistance used for the measurement of the voltage $y(t)=U(0, t)$ and current $u(t)=I(0, t)$, see Figure 1 . The energy dissipated in this resistor is re-created in the internal current source, to be dissipated again in the external load $R_{L}=\beta$. Thus, the net energy storage/dissipation inside the externally Cayley transformed system is due to the impedance $\hat{Z}(s)$ of the transmission line alone, and it is given by

$$
2 \int_{-\infty}^{T} y(t) u(t) d t=\int_{-\infty}^{T}\left(\left|u^{(\beta)}(t)\right|^{2}-\left|y^{(\beta)}(t)\right|^{2}\right) d t
$$

for any $T \in \mathbb{R}$ and $\beta>0$.

We next characterize impedance passivity or conservativity by means of a geometric condition in a particular Krĕ̌n space.

Theorem 3.3. Let $\Xi=\left(\left[\begin{array}{c}G \\ L \\ K\end{array}\right] ;\left[\begin{array}{l}\mathcal{U} \\ \mathcal{X}\end{array}\right]\right)$ be a colligation. 
(i) $\Xi$ is impedance passive if and only if its graph $\mathcal{G}(\Xi)$ is a maximal nonpositive subspace of of the Kre ̌n space $\mathfrak{K}_{i m p}$ induced by the signature operator $J_{\text {imp }}:=\left[\begin{array}{cccc}0 & 0 & 0 & -1 \\ 0 & 0 & 1 & 0 \\ 0 & 1 & 0 & 0 \\ -1 & 0 & 0 & 0\end{array}\right]$ in the Hilbert space $\left[\begin{array}{l}\mathcal{U} \\ \mathcal{X} \\ \mathcal{U}\end{array}\right]$.

(ii) $\Xi$ is impedance energy preserving if and only if its graph $\mathcal{G}(\Xi)$ is a neutral and maximal nonpositive subspace of the Krĕn space $\mathfrak{K}_{i m p}$ defined in (i).

(iii) $\Xi$ is impedance conservative if and only if its graph $\mathcal{G}(\Xi)$ is a Lagrangian subspace of the Krĕn space $\mathfrak{K}$ defined in (i).

In particular, the three words "some" in Definition 3.2 can be replaced by the word "all", without changing the meaning of the notions defined there.

Proof. For each $\beta \in \mathbb{C}^{+}$, the bijection $U_{\beta}^{\text {ext }}: \mathfrak{K}_{i m p} \rightarrow \mathfrak{K}_{s c}$ defined by

$$
U_{\beta}^{e x t}:=\left[\begin{array}{cccc}
\frac{1}{\sqrt{2 \operatorname{Re} \beta}} & 0 & 0 & 0 \\
0 & 1 & 0 & 0 \\
0 & 0 & 1 & 0 \\
0 & 0 & 0 & \frac{1}{\sqrt{2 \operatorname{Re} \beta}}
\end{array}\right]\left[\begin{array}{cccc}
\beta & 0 & 0 & 1 \\
0 & 1 & 0 & 0 \\
0 & 0 & 1 & 0 \\
\beta & 0 & 0 & -1
\end{array}\right]
$$

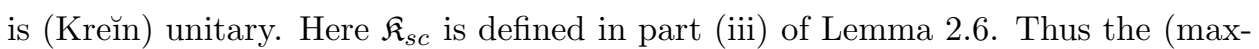
imal) nonpositivity, the (maximal) nonnegativity and neutrality of subspaces is preserved under this mapping. Since $U_{\beta}^{\text {ext }} \mathcal{G}(\Xi)=\mathcal{G}\left(\Xi^{(\beta)}\right)$, the claims (i)-(iii) follow from Lemmas 2.6 and 2.7.

Because none of the Krel̆n space conditions in claims (i)-(iii) of this lemma depends on the parameter $\beta$, we conclude that if any of these condition holds for one $\beta \in \mathbb{C}^{+}$, then the same condition holds for all $\beta \in \mathbb{C}^{+}$.

Theorem 3.4. Let $\Xi=\left(\left[\begin{array}{c}G \\ L \\ K\end{array}\right] ;\left[\begin{array}{l}\mathcal{U} \\ \mathcal{X} \\ \mathcal{U}\end{array}\right]\right)$ be a colligation.

(i) $\Xi$ is impedance passive if and only if the following two conditions hold:

(a) $\left[\begin{array}{c}\beta G+K \\ \alpha-L\end{array}\right]$ is surjective for some $\alpha, \beta \in \mathbb{C}^{+}$;

(b) For all $z \in \mathcal{D}$ om $(\Xi)$ we have

$$
\operatorname{Re}\langle z, L z\rangle_{\mathcal{X}} \leq \operatorname{Re}\langle K z, G z\rangle_{\mathcal{U}}
$$

(ii) $\Xi$ is impedance energy preserving if and only if it is impedance passive and (3.2) holds in the form of an equality, i.e, for all $z \in \mathcal{D}$ om $(\Xi)$ we have

$$
\operatorname{Re}\langle z, L z\rangle_{\mathcal{X}}=\operatorname{Re}\langle K z, G z\rangle_{\mathcal{U}}
$$

(iii) $\Xi$ is impedance conservative if and only if it is impedance energy preserving and, in addition,

(c) $\left[\begin{array}{c}\gamma-L \\ \beta G-K\end{array}\right]$ is surjective for some $\beta \in \mathbb{C}^{+}$and $\gamma \in \mathbb{C}^{-}$.

For an impedance passive $\Xi$, condition (a) holds for all $\alpha, \beta \in \mathbb{C}^{+}$. For an impedance conservative $\Xi$, also condition (c) holds for all $\beta \in \mathbb{C}^{+}$and $\gamma \in \mathbb{C}^{-}$.

Proof. All this follows from Lemmas 2.6 and 2.7, together with Theorem 3.3.

Remark 3.5. The cases $\operatorname{Re} \alpha=0$ and $\operatorname{Re} \gamma=0$ are excluded in Theorem 3.4 because the given conditions are no longer necessary for impedance passivity or conservativity in this case. However, conditions (a) and (c) are still sufficient for impedance passivity or conservativity even when $\operatorname{Re} \alpha=0$ or $\operatorname{Re} \gamma=0$. The proof of this fact is similar to that of Theorem 4.3. 
Impedance passive nodes also occur naturally in a different setting where the input and output spaces are different. In this case we need to reinterpret the righthand sides of (3.2) and (3.3) in a new way. Instead of requiring that $\mathcal{Y}=\mathcal{U}$ we now interpret $\mathcal{Y}$ as the dual space of $\mathcal{U}$. More precisely, we suppose that every bounded linear functional on $\mathcal{U}$ has a unique representation of the type $u \mapsto\langle y, u\rangle_{(\mathcal{Y}, \mathcal{U})}$ for some $y \in \mathcal{Y}$, where $\langle\cdot, \cdot\rangle_{(\mathcal{Y}, \mathcal{U})}$ is a duality pairing between $\mathcal{Y}$ and $\left.\mathcal{U}\right)$, and replace (3.2) and (3.3) by

$$
\operatorname{Re}\langle z, L z\rangle_{\mathcal{X}} \leq \operatorname{Re}\langle K z, G z\rangle_{(\mathcal{Y}, \mathcal{U})}
$$

and

$$
\operatorname{Re}\langle z, L z\rangle_{\mathcal{X}}=\operatorname{Re}\langle K z, G z\rangle_{(\mathcal{Y}, \mathcal{U})},
$$

respectively. By Riesz representation theorem, given a duality pairing $\langle\cdot, \cdot\rangle_{(\mathcal{Y}, \mathcal{U})}$ between $\mathcal{Y}$ and $\mathcal{U}$, there exists a unique unitary operator $\Psi: \mathcal{U} \rightarrow \mathcal{Y}$ such that

$$
\langle y, u\rangle_{(\mathcal{Y}, \mathcal{U})}=\langle y, \Psi u\rangle_{\mathcal{Y}}=\left\langle\Psi^{*} y, u\right\rangle_{\mathcal{U}}, \quad y \in \mathcal{Y}, \quad u \in \mathcal{U},
$$

and conversely, each unitary operator $\Psi: \mathcal{U} \rightarrow \mathcal{Y}$ defines a duality pairing between $\mathcal{Y}$ and $\mathcal{U}$ through (3.6). We shall refer to $\Psi$ as the duality map between $\mathcal{U}$ and $\mathcal{Y}$.

Definition 3.6. Let $\Xi=\left(\left[\begin{array}{l}G \\ L \\ K\end{array}\right] ;\left[\begin{array}{l}\mathcal{U} \\ \mathcal{X}\end{array}\right]\right)$ be a colligation, where $\mathcal{Y}$ has been identified with the dual of $\mathcal{U}$ with duality map $\Psi: \mathcal{U} \rightarrow \mathcal{Y}$. Then $\Xi$ is impedance passive (or energy preserving, or conservative) if the node $\Xi_{\Psi}=\left(\left[\begin{array}{c}G \\ L \\ \Psi^{*} K\end{array}\right] ;\left[\begin{array}{l}\mathcal{U} \\ \mathcal{U}\end{array}\right]\right)$ is passive (or energy preserving, or conservative) in the sense of Definition 3.2.

We leave it for an interested reader to formulate the modified versions of Theorems 3.3 and 3.4 for the case $\mathcal{U} \neq \mathcal{Y}$.

\section{INTERNAL WELL-POSEDNESS}

One of the most important problems for impedance passive colligations $\Xi$ is to verify that the associated Cauchy problems (1.1) have unique smooth solutions. This amounts to showing that $\Xi$ is an internally well-posed boundary node.

Proposition 4.1. An impedance passive colligation $\Xi=\left(\left[\begin{array}{c}G \\ L \\ K\end{array}\right] ;\left[\begin{array}{l}\mathcal{U} \\ \mathcal{X} \\ \mathcal{Y}\end{array}\right]\right)$ is an internally well-posed boundary node if and only if $\left[\begin{array}{c}G \\ \alpha-L\end{array}\right]$ is surjective for some $\alpha \in \mathbb{C}^{+}$. The semigroup of an impedance passive internally well-posed boundary node is a contraction semigroup.

In this proof, and in all other proofs in this section, we take for simplicity $\mathcal{Y}=\mathcal{U}$. To get the general case $\mathcal{Y} \neq \mathcal{U}$, it suffices to replace $K$ throughout by $\Psi^{*} K$ where $\Psi$ is the unitary operator in (3.6).

Proof of Proposition 4.1. The "only if" part is trivial. For the "if" part, note that $\left.L\right|_{\mathcal{N}(G)}$ generates a $C_{0}$ contraction semigroup by the argument given in the proof of Theorem 2.5, with (2.2) replaced by (3.4). The colligation $\Xi$ is closed, since $\mathcal{G}(\Xi)$ is closed as a maximal nonnegative subspace of the Krel̆n space $\mathfrak{K}_{i m p}$, see Theorem 3.3. Thus $\Xi$ is an internally well-posed boundary node.

The following proposition takes a closer look at the impedance conservative case. 
Proposition 4.2. Let $\Xi=\left(\left[\begin{array}{c}G \\ L \\ K\end{array}\right] ;\left[\begin{array}{l}\mathcal{U} \\ \mathcal{X} \\ \mathcal{Y}\end{array}\right]\right)$ be an impedance conservative colligation. Define $A:=\left.L\right|_{\mathcal{N}(G)}$ and $\Xi^{\prime}:=\left(\left[\begin{array}{c}G \\ -L \\ -K\end{array}\right] ;\left[\begin{array}{l}\mathcal{U} \\ \mathcal{X} \\ \mathcal{Y}\end{array}\right]\right)$. Then the colligation $\Xi^{\prime}$ is impedance conservative, too, and the following conditions are equivalent:

(i) $G$ is surjective, and both $\rho(A) \cap \mathbb{C}^{+} \neq \emptyset$ and $\rho(A) \cap \mathbb{C}^{-} \neq \emptyset$;

(ii) $G$ is surjective and $A^{*}=-A$;

(iii) $\Xi$ is an internally well-posed boundary node, and the semigroup of $\Xi$ is unitary;

(iv) Both $\Xi$ and $\Xi^{\prime}$ are internally well-posed boundary nodes.

In many PDE applications (including both the examples of Section 6 ) the operator $A=\left.L\right|_{\mathcal{N}(G)}$ has a compact resolvent. Then the intersection $\sigma(A) \cap i \mathbb{R}$ is at most countable, and the two spectral assumptions in (i) are satisfied.

Proof of Proposition 4.2. It is easy to check that $\mathcal{G}\left(\Xi^{\prime}\right)$ is Lagrangean if and only if $\mathcal{G}(\Xi)$ is Lagrangean. Thus the colligation $\Xi^{\prime}$ is impedance conservative by Theorem 3.3 .

The implication (i) $\Rightarrow$ (iv) follows from Proposition 4.1. By the same theorem, if (iv) holds, then both $A$ and $-A$ generate contraction semigroups, and hence the semigroups generated by these operators are unitary, so that (iii) holds. It is easy to see that (iii) $\Rightarrow$ (ii) $\Rightarrow$ (i).

The following theorem can be used to check both the impedance passivity (or conservativy) and the internal well-posedness at the same time:

Theorem 4.3. Let $\Xi=\left(\left[\begin{array}{c}G \\ L \\ K\end{array}\right] ;\left[\begin{array}{l}\mathcal{U} \\ \mathcal{X} \\ \mathcal{Y}\end{array}\right]\right)$ be a colligation with the property that $\left[\begin{array}{l}G \\ L\end{array}\right]$ is closed (with domain Dom $(\Xi)$ ) and $K$ is continuous with respect to the graph norm of $\left[\begin{array}{c}G \\ L\end{array}\right]$.

(i) Suppose that (3.4) holds for all $z \in \mathcal{D}$ om $(\Xi)$, and that $\left[\begin{array}{c}G \\ \alpha-L\end{array}\right]$ is surjective for some $\alpha \in \mathbb{C}$ with $\operatorname{Re} \alpha \geq 0$. Then $\Xi$ is an internally well-posed impedance passive boundary node.

(ii) If (3.5) holds for all $z \in \mathcal{D o m}(\Xi)$ and if both $\left[\begin{array}{c}G \\ \alpha-L\end{array}\right]$ and $\left[\begin{array}{c}G \\ \gamma-L\end{array}\right]$ are surjective for some $\alpha, \gamma \in \mathbb{C}$ with $\operatorname{Re} \alpha \geq 0$ and $\operatorname{Re} \gamma \leq 0$, then $\Xi$ is an internally well-posed impedance conservative boundary node which a unitary semigroup.

Proof. It is easy to see that $\Xi$ is closed (cf. Lemma 4.5).

According to part (i) of Theorem 3.4, in order to prove that $\Xi$ is impedance passive under the assumption of (i), it suffices to show that $\left[\begin{array}{c}G+\epsilon K \\ \alpha+\delta-L\end{array}\right]$ is surjective for all sufficiently small $\delta, \epsilon \in \mathbb{C}$.

Denote $\mathcal{H}:=\mathcal{N}\left(\left[\begin{array}{c}G \\ \alpha-L\end{array}\right]\right)^{\perp}$ where the orthogonal complement is taken in the graph inner product of $\operatorname{Dom}(\Xi)$. Both the operators $\left.\left[\begin{array}{c}G \\ \alpha-L\end{array}\right]\right|_{\mathcal{H}}$ and $\left.K\right|_{\mathcal{H}}$ map continuously $\mathcal{H} \mapsto\left[\begin{array}{c}\mathcal{U} \\ \mathcal{X}\end{array}\right]$. Since $\left[\begin{array}{c}G \\ \alpha-L\end{array}\right]$ is surjective, $\left[\begin{array}{c}G \\ \alpha-L\end{array}\right] \mid \mathcal{H}$ is boundedly invertible. Because the set of boundedly invertible operators is open, the operator $\left.\left[\begin{array}{c}G+\epsilon K \\ \delta+\alpha-L\end{array}\right]\right|_{\mathcal{H}}=\left.\left[\begin{array}{c}G \\ \alpha-L\end{array}\right]\right|_{\mathcal{H}}+\left.\left[\begin{array}{c}\epsilon K \\ \delta\end{array}\right]\right|_{\mathcal{H}}$ is boundedly invertible for all $\delta, \epsilon \in \mathbb{C}$ small enough. In particular, $\left[\begin{array}{c}G+\epsilon K \\ \delta+\alpha-L\end{array}\right]$ maps $\mathcal{D}$ om $(\Xi)$ onto $\left[\begin{array}{c}\mathcal{X} \\ \mathcal{X}\end{array}\right]$. By Theorem $3.4, \Xi$ is impedance passive. By Proposition 4.1, $\Xi$ is an internally well-posed boundary node.

The same proof shows that (ii) holds, too (replace part (i) of Theorem 3.4 by part (ii), and replace Proposition 4.1 by Proposition 4.2). 
Many PDE examples satisfy even stronger conditions than those of Theorem 4.3:

Definition 4.4. A colligation $\Xi=\left(\left[\begin{array}{c}G \\ L \\ K\end{array}\right] ;\left[\begin{array}{c}\mathcal{U} \\ \mathcal{X} \\ \mathcal{Y}\end{array}\right]\right)$ is strong if it is closed and its interior operator $L$ is closed with $\operatorname{Dom}(L)=\operatorname{Dom}(\Xi)$.

We remark that an impedance or scattering conservative internally well-posed boundary node need not be strong, see Section 6.2 .

Lemma 4.5. A colligation $\Xi=\left(\left[\begin{array}{l}G \\ L \\ K\end{array}\right] ;\left[\begin{array}{l}\mathcal{U} \\ \mathcal{X} \\ \mathcal{Y}\end{array}\right]\right)$ is strong if and only if its interior operator $L$ is closed with $\operatorname{Dom}(L)=\mathcal{D}$ om $(\Xi)$, and $G$ and $K$ are continuous with respect to the graph norm of $L$ on $\mathcal{D}$ om $(\Xi)$.

Proof. Suppose that $\Xi$ is strong. Then we get two different graph norms on $\operatorname{Dom}(\Xi)$, namely the graph norm of $L$ and the graph norm of $\left[\begin{array}{l}G \\ L \\ K\end{array}\right]$. The former is clearly weaker than the latter, and hence by the closed graph theorem, they are equivalent. This implies that $G$ and $K$ are continuous with respect to the graph norm of $L$. The easy proof of the converse claim that the continuity of $G$ and $K$ with respect to the graph norm of $L$ implies that $\Xi$ is closed is left to the reader.

Remark 4.6. The general assumption in Theorem 4.3 about the closedness of $\left[\begin{array}{c}G \\ L\end{array}\right]$ and the continuity of $K$ with respect to the graph norm of $\left[\begin{array}{c}G \\ L\end{array}\right]$ holds whenever $\Xi$ is a strong colligation.

Theorem 4.7. An impedance passive strong colligation is an internally well-posed boundary node if and only if its input boundary operator $G$ is surjective.

Proof. We denote the colligation by $\Xi=\left(\left[\begin{array}{c}G \\ L \\ K\end{array}\right] ;\left[\begin{array}{l}\mathcal{U} \\ \mathcal{X} \\ \mathcal{Y}\end{array}\right]\right)$. By Proposition 4.1 , it is enough to show that $\alpha-A$ is surjective for some $\alpha>0$, where $A:=\left.L\right|_{\mathcal{N}(G)}$.

Let $\alpha>0$. By Theorem 3.4, $\left[\begin{array}{c}\beta G+K \\ \alpha-L\end{array}\right]$ is surjective for all $\beta \in \mathbb{C}^{+}$. This implies that $\left[\begin{array}{c}G+\epsilon K \\ \alpha-L\end{array}\right]$ is surjective for all $\epsilon>0$. Let $x \in \mathcal{X}$ be arbitrary, and let $z_{\epsilon} \epsilon$ $\operatorname{Dom}(\Xi)=\operatorname{Dom}(L)$ be any solution of the equation

$$
\left[\begin{array}{c}
G+\epsilon K \\
\alpha-L
\end{array}\right] z_{\epsilon}=\left[\begin{array}{l}
0 \\
x
\end{array}\right] .
$$

Then it follows from (3.2) that $\alpha\left\|z_{\epsilon}\right\|_{\mathcal{X}} \leq\|x\|_{\mathcal{X}}$. Clearly

$$
\left\|L z_{\epsilon}\right\|_{\mathcal{X}}=\left\|\alpha z_{\epsilon}-x\right\|_{\mathcal{X}} \leq \alpha\left\|z_{\epsilon}\right\|_{\mathcal{X}}+\|x\|_{\mathcal{X}} \leq 2\|x\|_{\mathcal{X}} .
$$

It follows that there exists exactly one solution $z_{\epsilon}$ of (4.1) for any given $x$. Furthermore, $z_{\epsilon}$ is bounded in the graph norm of $L$, uniformly in $\epsilon>0$.

Let $\mathcal{Z}$ be the Hilbert space $\operatorname{Dom}(L)$ equipped with the graph norm of $L$. The unit ball in $\mathcal{Z}$ is weakly sequentially compact, and therefore there exists a sequence $\epsilon_{n} \rightarrow 0$ such that $z_{\epsilon_{n}} \rightarrow z$ weakly in $\mathcal{Z}$ as $n \rightarrow \infty$. Both $G$ and $K$ are continuous from $\mathcal{Z}$ to $\mathcal{U}$, hence weakly continuous. Thus, $G z_{\epsilon_{n}} \rightarrow G z$ and $K z_{\epsilon_{n}} \rightarrow K z$ weakly in $\mathcal{U}$. Substituting this into (4.1) we find that $z \in \mathcal{N}(G)$ and $(\alpha-L) z=(\alpha-A) z=$ $x$, proving the surjectivity of $\alpha-A$.

Corollary 4.8. Let $\Xi=\left(\left[\begin{array}{l}G \\ L \\ K\end{array}\right] ;\left[\begin{array}{l}\mathcal{U} \\ \mathcal{X} \\ \mathcal{Y}\end{array}\right]\right)$ be an impedance conservative strong colligation. If $\mathcal{R}(G)=\mathcal{U}$, then both $\left(\left[\begin{array}{l}G \\ L \\ K\end{array}\right] ;\left[\begin{array}{l}\mathcal{U} \\ \mathcal{X} \\ \mathcal{Y}\end{array}\right]\right)$ and $\left(\left[\begin{array}{c}G \\ -L \\ -K\end{array}\right] ;\left[\begin{array}{l}\mathcal{U} \\ \mathcal{Y}\end{array}\right]\right)$ are internally well-posed impedance conservative boundary nodes with unitary semi-groups. 
If $\mathcal{R}(K)=\mathcal{Y}$, then both $\left(\left[\begin{array}{l}K \\ L \\ G\end{array}\right] ;\left[\begin{array}{c}\mathcal{Y} \\ \mathcal{X} \\ \mathcal{U}\end{array}\right]\right)$ and $\left(\left[\begin{array}{c}K \\ -L \\ -G\end{array}\right] ;\left[\begin{array}{c}\mathcal{Y} \\ \mathcal{X} \\ \mathcal{U}\end{array}\right]\right)$ are internally wellposed conservative boundary nodes with unitary semi-groups. If $\mathcal{R}(G)=\mathcal{U}$ and $\mathcal{R}(K)=\mathcal{Y}$, then $\Xi$ is a time-flow invertible boundary node in the sense of [MS06a, Definition 1.3].

This follows directly from Proposition 4.2 and Theorem 4.7.

\section{Abstract boundary SPACES FOR SYMMETRIC OPERATORS}

It is a classical problem in operator theory to parameterize self-adjoint extensions of a given closed, densely defined symmetric operator $\mathcal{A}$ on Hilbert space $\mathcal{X}$. The results are often formulated in terms of abstract boundary spaces for $\mathcal{A}$. It turns out that this notion is a special case of our notion of impedance conservative strong bondary node.

We shall study extensions of the operator

$$
L_{0}:=\left.L\right|_{\mathcal{D o m}\left(L_{0}\right)} \quad \text { with } \quad \mathcal{D} o m\left(L_{0}\right):=\mathcal{N}(G) \cap \mathcal{N}(K)
$$

where $L$ is taken from an impedance passive colligation $\Xi=\left(\left[\begin{array}{c}G \\ L \\ K\end{array}\right] ;\left[\begin{array}{c}\mathcal{U} \\ \mathcal{X} \\ \mathcal{U}\end{array}\right]\right)$. Such an operator $L_{0}$ is called the minimal operator of $\Xi$. If $\Xi$ is impedance passive, it follows from (3.2) that $L_{0}$ is symmetric, and therefore at least closable. If $\Xi$ is strong, then $L_{0}$ is closed. The following definition is taken from [GG91].

Definition 5.1. Let $\mathcal{A}$ be a closed, densely defined symmetric operator on $\mathcal{X}$. The triple $\left(\mathcal{U}, \Gamma_{1}, \Gamma_{2}\right)$ is an abstract boundary space for $\mathcal{A}$ if $\mathcal{U}$ is a Hilbert space and $\Gamma_{1}, \Gamma_{2}$ are linear mappings from $\mathcal{D o m}\left(\mathcal{A}^{*}\right)$ into $\mathcal{U}$ with the following properties:

(i) for any $x, z \in \mathcal{D}$ om $\left(\mathcal{A}^{*}\right)$ we have

$$
\left\langle\mathcal{A}^{*} x, z\right\rangle_{\mathcal{X}}-\left\langle x, \mathcal{A}^{*} z\right\rangle_{\mathcal{X}}=\left\langle\Gamma_{1} x, \Gamma_{2} z\right\rangle_{\mathcal{U}}-\left\langle\Gamma_{2} x, \Gamma_{1} z\right\rangle_{\mathcal{U}}
$$

(ii) the mapping $\left[\begin{array}{l}\Gamma_{1} \\ \Gamma_{2}\end{array}\right]$ from $\operatorname{Dom}\left(\mathcal{A}^{*}\right)$ into $\left[\begin{array}{l}\mathcal{U} \\ \mathcal{U}\end{array}\right]$ is surjective.

Theorem 5.2. If $\Xi=\left(\left[\begin{array}{l}G \\ L \\ K\end{array}\right] ;\left[\begin{array}{l}\mathcal{U} \\ \mathcal{X}\end{array}\right]\right)$ is an impedance conservative strong colligation and $\left[\begin{array}{c}G \\ K\end{array}\right]$ is surjective, then the operator $\mathcal{A}:=i L_{0}$ (where $L_{0}$ is defined by (5.1)) is closed, densely defined and symmetric, and the triple $(\mathcal{U}, G, i K)$ is an abstract boundary space for $\mathcal{A}$.

Conversely, if $\mathcal{A}$ is a closed, densely defined symmetric operator on $\mathcal{X}$ such that the triple $\left(\mathcal{U}, \Gamma_{1}, \Gamma_{2}\right)$ is an abstract boundary space for $\mathcal{A}$, then $\Xi=\left(\left[\begin{array}{c}\Gamma_{1} \\ i \mathcal{A}^{*} \\ -i \Gamma_{2}\end{array}\right] ;\left[\begin{array}{l}\mathcal{U} \\ \mathcal{X} \\ \mathcal{U}\end{array}\right]\right)$ is an impedance conservative internally well-posed strong boundary node with a unitary semigroup. This node has all the properties listed in Corollary 4.8.

Proof. We first prove the direct part, and begin by noticing that if $\Xi=\left(\left[\begin{array}{c}G \\ L \\ K\end{array}\right] ;\left[\begin{array}{c}\mathcal{U} \\ \mathcal{X} \\ \mathcal{U}\end{array}\right]\right)$ is an impedance conservative strong colligation and $\left[\begin{array}{c}G \\ K\end{array}\right]$ is surjective, then all the conclusions listed in Corollary 4.8 hold.

By polarizing (3.3) we get

(5.2) $\langle L x, z\rangle_{\mathcal{X}}=\langle x,-L z\rangle_{\mathcal{X}}+\langle K x, G z\rangle_{\mathcal{U}}+\langle K x, G z\rangle_{\mathcal{U}}$, for all $x, z \in \mathcal{D o m}(L)$.

If $z \in \operatorname{Dom}\left(L_{0}\right)$, then the right-hand side reduces to $-\left\langle x, L_{0} z\right\rangle \mathcal{X}$, and we conclude the inclusion $L_{0} \subset-L^{*}$. The operator $A=\left.L\right|_{\mathcal{N}(G)}$ satisfies $A \subset L$, and hence $L^{*} \subset$ $A^{*}$. Thus $L_{0} \subset-L^{*} \subset-A^{*}$, and hence $\mathcal{N}(G) \cap \mathcal{N}(K) \subset \mathcal{D o m}\left(L^{*}\right) \subset \mathcal{D o m}\left(A^{*}\right)$. 
By Corollary 4.8, we have $A^{*}=-A$ and thus $\mathcal{D}$ om $\left(A^{*}\right)=\mathcal{N}(G)$. It follows that $\mathcal{N}(G) \cap \mathcal{N}(K) \subset \mathcal{D}$ om $\left(L^{*}\right) \subset \mathcal{N}(G)$. The same argument with $\Xi$ replaced by the node $\left(\left[\begin{array}{c}K \\ L \\ G\end{array}\right] ;\left[\begin{array}{l}\mathcal{U} \\ \mathcal{X}\end{array}\right]\right)$ implies that $\mathcal{N}(G) \cap \mathcal{N}(K) \subset \mathcal{D}$ om $\left(L^{*}\right) \subset \mathcal{N}(K)$ and hence $\mathcal{D} o m\left(L^{*}\right)=\mathcal{D}$ om $\left(L_{0}\right)$. Because $L_{0} \subset-L^{*}$, we conclude that $L_{0}=-L^{*}$. Thus $L_{0}$ is closed and densely defined. Define $\mathcal{A}:=i L_{0}$. Then $\mathcal{A}$ is closed, densely defined, and symmetic, and it follows from $(5.2)$ that $(\mathcal{U}, G, i K)$ is an abstract boundary space for $\mathcal{A}$.

For the converse part we assume that $\mathcal{A}$ is a closed, densely defined symmetric operator on $\mathcal{X}$ such that the triple $\left(\mathcal{U}, \Gamma_{1}, \Gamma_{2}\right)$ is an abstract boundary space for $\mathcal{A}$. By [GG91, Theorem 1.6 p. 156] (with $K=0$ ), both the operators $\left.\mathcal{A}^{*}\right|_{\mathcal{N}\left(\Gamma_{1}-i \Gamma_{2}\right)}$ and $-\left.\mathcal{A}^{*}\right|_{\mathcal{N}\left(\Gamma_{1}+i \Gamma_{2}\right)}$ are maximal dissipative. In particular, their resolvent sets contain $\mathbb{C}^{+}$. By [GG91, Lemma 3.1 p. 164], the operators $\Gamma_{1}$ and $\Gamma_{2}$ are continuous $\mathcal{D o m}\left(\mathcal{A}^{*}\right) \rightarrow \mathcal{U}$. Clearly, both $\Gamma_{1}-i \Gamma_{2}$ and $\Gamma_{1}+i \Gamma_{2}$ are surjective. Thus both $\left[\begin{array}{c}\Gamma_{1}-i \Gamma_{2} \\ \alpha-i \mathcal{A}^{*}\end{array}\right]$ and $\left[\begin{array}{c}\Gamma_{1}+i \Gamma_{2} \\ \alpha+i \mathcal{A}^{*}\end{array}\right]$ are surjective for all $\alpha \in \mathbb{C}^{+}$. As part (i) of Definition 5.1 implies (3.3) with $G=\Gamma_{1}, L=i \mathcal{A}^{*}$ and $K=-i \Gamma_{2}$, we conclude from Theorem 3.4 that the colligation $\Xi$ is impedance conservative with $\operatorname{Dom}(\Xi)=\mathcal{D}$ om $\left(\mathcal{A}^{*}\right)$. Since $L$ is closed, it is a strong colligation. The remaining claims follow from Corollary 4.8 .

\section{Two Examples}

6.1. The transmission line. We now complete the study of the (impedance) transmission line equations (1.2) using the results of this paper. We define the operators $G, L$ and $K$ as follows:

$$
\begin{aligned}
L & :=\left[\begin{array}{cc}
0 & -\frac{1}{L(\xi)} \frac{\partial}{\partial \xi} \\
-\frac{1}{C(\xi)} \frac{\partial}{\partial \xi} & 0
\end{array}\right], \\
G & :=\left.\left[\begin{array}{ll}
\gamma_{0} & 0
\end{array}\right]\right|_{\mathcal{Z}}, \quad \text { and } \quad K:=\left.\left[\begin{array}{ll}
0 & \gamma_{0}
\end{array}\right]\right|_{\mathcal{Z}}
\end{aligned}
$$

where $\gamma_{0} f=f(0)$ is the Dirichlet trace for $f \in H^{1}(0,1)$, and let

$$
\mathcal{X}:=\left[\begin{array}{l}
L^{2}(0,1) \\
L^{2}(0,1)
\end{array}\right] \quad \text { and } \quad \mathcal{Z}:=\left\{\left[\begin{array}{l}
z_{1} \\
z_{2}
\end{array}\right] \in\left[\begin{array}{l}
H^{1}(0,1) \\
H^{1}(0,1)
\end{array}\right]: z_{1}(1)=0\right\} .
$$

We use the energy norm $\left\|\left[\begin{array}{c}z_{1} \\ z_{2}\end{array}\right]\right\|^{2}=\int_{0}^{1}\left(L(\xi)\left|z_{1}(\xi)\right|^{2}+C(\xi)\left|z_{2}(\xi)\right|^{2}\right) d \xi$ in $\mathcal{X}$. It is easy to see that $\Xi=\left(\left[\begin{array}{l}G \\ L \\ K\end{array}\right] ;\left[\begin{array}{l}\mathbb{C} \\ \mathbb{X}\end{array}\right]\right)$ is a strong colligation with $\mathcal{D}$ om $(\Xi)=\mathcal{Z}$. If $z(t)=\left[\begin{array}{c}I(\cdot, t) \\ U(\cdot, t)\end{array}\right] \in \mathcal{X}$ denotes the joint current/voltage distribution at time $t \geq 0$, then equations (1.2) take the form of (1.1).

Proposition 6.1. The strong colligation $\Xi$ defined above is an impedance conservative boundary node which has all the additional properties listed in Corollary 4.8. The operator $L_{0}$ given by (5.1) is closed and densely defined, and $(\mathbb{C}, G, i K)$ is an abstract boundary space for the operator $\mathcal{A}:=i L_{0}$.

Proof. It follows by partial integration that the Green-Lagrange identity (3.3) holds for all $z \in \mathcal{Z}$ when the inner product associated to the energy norm is used for $\mathcal{X}$. It is trivial that $G+K=\left[\begin{array}{ll}\gamma_{0} & \gamma_{0}\end{array}\right]$ maps $\mathcal{Z}$ onto input space $\mathbb{C}$.

We show next that $L$ maps $\mathcal{N}(G+K)$ onto $\mathcal{X}$. For any $\left[\begin{array}{l}x_{1} \\ x_{2}\end{array}\right] \in \mathcal{X}$ we have $L\left[\begin{array}{l}z_{1} \\ z_{2}\end{array}\right]=\left[\begin{array}{l}x_{1} \\ x_{2}\end{array}\right]$ for all $z_{1}, z_{2} \in H^{1}(0,1)$ that satisfy $z_{1}(x)-z_{1}(0)=-\int_{0}^{x} C(v) x_{2}(v) d v$ 
and $z_{2}(x)-z_{2}(0)=-\int_{0}^{x} L(v) x_{1}(v) d v$. It is possible choose the integration constants $z_{1}(0)$ and $z_{2}(0)$ so that $z_{1}(1)=0$ and $z_{1}(0)+z_{2}(0)=0$. Then $\left[\begin{array}{l}z_{1} \\ z_{2}\end{array}\right] \in$ $\mathcal{N}(G+K)$ and we conclude that $L$ maps $\mathcal{N}(G+K)$ onto $\mathcal{X}$. It is easy to see that $\mathcal{N}(L) \cap \mathcal{N}(G+K)=\{0\}$. It follows that $0 \in \rho\left(\left.L\right|_{\mathcal{N}(G+K)}\right)$ and hence there exists an $\alpha>0$ such that condition (a) of Theorem 3.4 holds with $\beta=1$. The remaining condition (c) of Theorem 3.4 is verified analogously, and we conclude that $\Xi$ is an impedance conservative strong colligation.

Since the operator $\left[\begin{array}{l}G \\ K\end{array}\right]$ is trivially surjective, the rest of the claims follow from Corollary 4.8 and Theorems 4.7 and 5.2.

In particular, we conclude that (1.2) has a smooth solution as explained after Definition 2.1 (when it is interpreted as equations in (1.1) by using (6.1) and (6.2)).

6.2. The wave equation. A more advanced example is provided by the wave equation with Neumann boundary control. We consider the linear system described by

$$
\left\{\begin{array}{l}
z_{t t}(t, \xi)=\Delta z(t, \xi) \quad \text { for } \xi \in \Omega \text { and } t \geq 0, \\
u(t, \xi)=\frac{\partial z}{\partial \nu}(t, \xi) \quad \text { for } \xi \in \Gamma_{1} \text { and } t \geq 0, \\
y(t, \xi)=z_{t}(t, \xi) \quad \text { for } \xi \in \Gamma_{1} \text { and } t \geq 0, \\
z(t, \xi)=0 \quad \text { for } \xi \in \Gamma_{0} \text { and } t \geq 0, \text { and } \\
z(0, \xi)=z_{0}(\xi), \quad z_{t}(0, \xi)=w_{0}(\xi) \quad \text { for } \xi \in \Omega .
\end{array}\right.
$$

Here $n \geq 2$ and $\Omega \subset \mathbb{R}^{n}$ is a bounded domain (open connected set) with $C^{2}$ boundary $\partial \Omega$. We assume that $\partial \Omega=\Gamma_{0} \cup \Gamma_{1}$ with $\overline{\Gamma_{0}} \cap \overline{\Gamma_{1}}=\emptyset$ where both $\Gamma_{0}$ and $\Gamma_{1}$ are nonempty. Thus $\Omega$ cannot be simply connected.

To rewrite this system as a boundary node we introduce the standard Sobolev spaces $H^{s}(\Omega), s=1,3 / 2,2$, and the boundary spaces $H^{s}(\Gamma), s= \pm 1 / 2$, which can be defined in several different ways (see [MS06a] for a short dicussion on this and note that $H^{0}=L^{2}$ ). The Dirichlet trace operator $\gamma$ is first defined for functions $f \in C^{\infty}(\bar{\Omega})$ by setting $\gamma f:=\left.f\right|_{\partial \Omega}$. This operator has a unique extension to an operator (still denoted by $\gamma$ ) which maps $H^{1}(\Omega)$ continuously onto $H^{1 / 2}(\partial \Omega)$. Let

$$
H_{\Gamma_{0}}^{1}(\Omega)=\left\{f \in H^{1}(\Omega):\left.f\right|_{\Gamma_{0}}=0\right\}
$$

where we write $\gamma f:=\left.f\right|_{\partial \Omega}$. Then the operator $\gamma_{0}:=\left.\gamma\right|_{H_{\Gamma_{0}}^{1}(\Omega)} \operatorname{maps} H_{\Gamma_{0}}^{1}(\Omega)$ continuously onto $H^{1 / 2}\left(\Gamma_{1}\right)$, and we abbreviate $\gamma_{0} f=\left.f\right|_{\Gamma_{1}}$.

The Neumann trace operator $\gamma \frac{\partial}{\partial \nu}$ is first defined on $C^{\infty}(\bar{\Omega})$ (with values in $\left.L^{2}(\partial \Omega)\right)$ by setting $\left(\gamma \frac{\partial}{\partial \nu} f\right)(\xi):=\nu(\xi) \cdot \nabla f(\xi)$ for all $\xi \in \partial \Omega$ where $\nu(\xi)$ denotes the outward unit normal vector of $\partial \Omega$ at $\xi$. Following [Gri85, p. 59], define the Hilbert space

$$
E\left(\Delta ; L^{2}(\Omega)\right):=\left\{f \in H^{1}(\Omega): \Delta f \in L^{2}(\Omega)\right\}
$$

with the norm $\|f\|_{E\left(\Delta ; L^{2}(\Omega)\right)}^{2}=\|f\|_{H^{1}(\Omega)}^{2}+\|\Delta f\|_{L^{2}(\Omega)}^{2}$. The operator $\gamma \frac{\partial}{\partial \nu}$ has a unique extension to an operator which maps $E\left(\Delta ; L^{2}(\Omega)\right)$ continuously into $H^{-1 / 2}(\partial \Omega)$; the proof given in [LM72, Theorem 6.5 on p. 175] assumes $C^{\infty}$ boundary for $\Omega$ but the same argument is valid for $C^{2}$ boundaries, too. We shall make use of the following closed subspace

$$
\mathcal{Z}_{0}:=\left\{f \in H_{\Gamma_{0}}^{1}(\Omega): \Delta f \in L^{2}(\Omega)\right\}
$$


of $E\left(\Delta ; L^{2}(\Omega)\right)$, and it is equipped with the same norm as $E\left(\Delta ; L^{2}(\Omega)\right)$. Since the Neumann problem

$$
\Delta z_{0}=0,\left.\quad z_{0}\right|_{\Gamma_{0}}=0,\left.\quad \frac{\partial z_{0}}{\partial \nu}\right|_{\Gamma_{1}}=u
$$

has always a variational solution in $H^{1}(\Omega)$ for all $u \in H^{-1 / 2}\left(\Gamma_{1}\right)$, we see that $\gamma \frac{\partial}{\partial \nu}$ maps $\mathcal{Z}_{0}$ onto $H^{-1 / 2}\left(\Gamma_{1}\right)$. We abbreviate the Neumann trace operator on $\mathcal{Z}_{0}$ as $\left(\gamma \frac{\partial}{\partial \nu}\right) f=\left.\frac{\partial f}{\partial \nu}\right|_{\Gamma_{1}}$. See [MS06a] for further details and references on Sobolev spaces and trace mappings.

After these preparations, let us return to equations (6.3). We obtain first order equations of form (1.1) by noting that $z_{t t}=\Delta z$ is equivalent to the first order equation $\frac{d}{d t}\left[\begin{array}{c}z \\ w\end{array}\right]=\left[\begin{array}{ll}0 & 1 \\ \Delta & 0\end{array}\right]\left[\begin{array}{c}z \\ w\end{array}\right]$. The spaces $\mathcal{Z}, \mathcal{X}$ and and operator $L$ are defined by

$$
\begin{gathered}
L:=\left[\begin{array}{ll}
0 & 1 \\
\Delta & 0
\end{array}\right]: \mathcal{Z} \rightarrow \mathcal{X} \text { with } \\
\mathcal{Z}:=\mathcal{Z}_{0} \times H_{\Gamma_{0}}^{1}(\Omega) \text { and } \mathcal{X}:=H_{\Gamma_{0}}^{1}(\Omega) \times L^{2}(\Omega)
\end{gathered}
$$

where $H_{\Gamma_{0}}^{1}(\Omega)$ and $\mathcal{Z}_{0}$ are given by (6.4) and (6.6), respectively. For the space $\mathcal{X}$, we use the energy norm

$$
\left\|\left[\begin{array}{c}
z_{0} \\
w_{0}
\end{array}\right]\right\|_{\mathcal{X}}^{2}:=\left\|\nabla z_{0}\right\|_{L^{2}(\Omega)}^{2}+\left\|w_{0}\right\|_{L^{2}(\Omega)}^{2} .
$$

Defining $\mathcal{U}:=H^{-1 / 2}\left(\Gamma_{1}\right), G\left[\begin{array}{c}z_{0} \\ w_{0}\end{array}\right]:=\left.\frac{\partial z_{0}}{\partial \nu}\right|_{\Gamma_{1}}, \mathcal{Y}:=H^{1 / 2}\left(\Gamma_{1}\right)$, and $K\left[\begin{array}{c}z_{0} \\ w_{0}\end{array}\right]:=\left.w_{0}\right|_{\Gamma_{1}}$, we obtain the colligation $\Xi=\left(\left[\begin{array}{l}G \\ L \\ K\end{array}\right] ;\left[\begin{array}{l}\mathcal{U} \\ \mathcal{Y}\end{array}\right]\right)$.

Proposition 6.2. The colligation $\Xi$ defined above is an impedance conservative strong boundary node with respect to the standard duality pairing between $\mathcal{U}=$ $H^{-1 / 2}\left(\Gamma_{1}\right)$ and $\mathcal{Y}=H^{1 / 2}\left(\Gamma_{1}\right)$ with pivot space $L^{2}\left(\Gamma_{1}\right)$. This node has all the additional properties listed in Corollary 4.8.

Moreover, the operator $L_{0}$ given by (5.1) is closed and densely defined, and $\left(\mathcal{U}, G, i \Psi^{*} K\right)$ is an abstract boundary space for the operator $\mathcal{A}:=i L_{0}$, where $\Psi$ is the duality map between $\mathcal{U}$ and $\mathcal{Y}$.

In particular, the equation (6.3) has a smooth solution (as explained after Definition 2.1) when it is interpreted as equations (1.1) by using the above definitions.

Proof. It is easy to see that $T:=\left[\begin{array}{ll}0 & 1 \\ \Delta & 0\end{array}\right]$ is a closed operator in $\mathcal{X}$ with domain $\operatorname{Dom}(T):=E\left(\Delta ; L^{2}(\Omega)\right) \times H_{\Gamma_{0}}^{1}(\Omega)$. Since the Dirichlet trace maps continuously $E\left(\Delta ; L^{2}(\Omega)\right) \rightarrow L^{2}\left(\Gamma_{0}\right)$, it follows from Lemma 6.6 that $L$ is closed with $\mathcal{D} o m(L)=$ $\mathcal{Z}$.

For an arbitrary $\left[\begin{array}{c}z_{0} \\ w_{0}\end{array}\right] \in \mathcal{Z}$, the Green's formula [Gri85, p. 62] implies the GreenLagrange identity (3.5) since

$$
\begin{aligned}
& 2 \operatorname{Re}\left\langle\left[\begin{array}{c}
z_{0} \\
w_{0}
\end{array}\right], L\left[\begin{array}{c}
z_{0} \\
w_{0}
\end{array}\right]\right\rangle_{\mathcal{X}}=2 \operatorname{Re}\left\langle\left[\begin{array}{c}
z_{0} \\
w_{0}
\end{array}\right],\left[\begin{array}{c}
w_{0} \\
\Delta z_{0}
\end{array}\right]\right\rangle_{\mathcal{X}} \\
& =2 \operatorname{Re}\left(\left\langle\Delta \overline{z_{0}}, w_{0}\right\rangle_{L^{2}(\Omega)}+\int_{\Omega} \nabla \overline{z_{0}} \cdot \nabla w_{0} d \Omega\right)=2 \operatorname{Re}\left(\int_{\Gamma_{0} \cup \Gamma_{1}} \frac{\partial \overline{z_{0}}}{\partial \nu} w_{0} d \omega\right) \\
& =2 \operatorname{Re}\left\langle\left.\frac{\partial z_{0}}{\partial \nu}\right|_{\Gamma_{1}},\left.w_{0}\right|_{\Gamma_{1}}\right\rangle_{\left(H^{-1 / 2}\left(\Gamma_{1}\right) ; H^{1 / 2}\left(\Gamma_{1}\right)\right)}=2 \operatorname{Re}\left\langle G\left[\begin{array}{c}
z_{0} \\
w_{0}
\end{array}\right], K\left[\begin{array}{c}
z_{0} \\
w_{0}
\end{array}\right]\right\rangle_{(\mathcal{U} ; \mathcal{Y})}
\end{aligned}
$$

where we have used $\left.w_{0}\right|_{\Gamma_{0}}=0$. 
Let $u \in \mathcal{U}$ and $\left[\begin{array}{c}z_{0} \\ w_{0}\end{array}\right] \in \mathcal{X}$ be arbitrary. We have now $\left[\begin{array}{l}G \\ L\end{array}\right]\left[\begin{array}{l}x_{0} \\ v_{0}\end{array}\right]=\left[\begin{array}{c}u \\ z_{0} \\ w_{0}\end{array}\right]$ for $\left[\begin{array}{l}x_{0} \\ v_{0}\end{array}\right] \in \mathcal{Z}$ if and only if $v_{0}=z_{0}$ and $x_{0}$ is a solution in $H^{1}(\Omega)$ of the problem

$$
\Delta x_{0}=w_{0},\left.\quad x_{0}\right|_{\Gamma_{0}}=0,\left.\quad \frac{\partial x_{0}}{\partial \nu}\right|_{\Gamma_{1}}=u .
$$

But this problem has a unique variational solution ${ }^{3}$ in $H^{1}(\Omega)$ because $w_{0} \in L^{2}(\Omega)$ and $u \in L^{2}\left(\Gamma_{1}\right)$. We conclude that $\left[\begin{array}{l}G \\ L\end{array}\right]$ maps $\mathcal{Z}$ onto $\left[\begin{array}{l}\mathcal{U} \\ \mathcal{X}\end{array}\right]$. It now follows from Theorem 4.3 that $\Xi$ is an internally well-posed, impedance passive boundary node.

We show next that $\left[\begin{array}{c}G \\ K\end{array}\right] \operatorname{maps} \mathcal{Z}$ onto $\left[\begin{array}{l}\mathcal{U} \\ \mathcal{Y}\end{array}\right]$; i.e., that there exists a $z_{0} \in \mathcal{Z}_{0}$ and $w_{0} \in H_{\Gamma_{0}}^{1}(\Omega)$ such that $\left[\begin{array}{l}G \\ K\end{array}\right]\left[\begin{array}{c}z_{0} \\ w_{0}\end{array}\right]=\left[\begin{array}{c}\left.\frac{\partial z_{0}}{\partial \nu}\right|_{\Gamma_{1}} \\ \left.w_{0}\right|_{\Gamma_{1}}\end{array}\right]=\left[\begin{array}{l}u \\ y\end{array}\right]$ for all $u \in H^{-1 / 2}\left(\Gamma_{0}\right)$ and $y \in H^{1 / 2}\left(\Gamma_{0}\right)$. This follows by choosing $z_{0}$ as the unique variational solution in $H^{1}(\Omega)$ of $(6.7)$ and $w_{0}$ as the unique variational solution in $H^{1}(\Omega)$ of

$$
\Delta w_{0}=0,\left.\quad w_{0}\right|_{\Gamma_{0}}=0,\left.\quad w_{0}\right|_{\Gamma_{1}}=y .
$$

Now the rest of the claims follow from Corollary 4.8 and Theorems 4.7 and 5.2.

The wave equation problem (6.3) can be interpreted as an impedance conservative internally well-posed boundary node also in another setting, where the input space is equal to the output space, namely $\mathcal{U}=\mathcal{Y}=L^{2}\left(\Gamma_{1}\right)$. We get this node simply by restricting the domain of the node $\Xi$ in Proposition 6.2 to the subspace

$$
\mathcal{Z}^{\prime}=\left\{\left[\begin{array}{c}
z_{0} \\
w_{0}
\end{array}\right] \in \mathcal{Z}: G z_{0} \in L^{2}\left(\Gamma_{1}\right)\right\}
$$

We remark that this space was denoted by $\mathcal{Z}$ in [MS06a, Section 5].

Proposition 6.3. Let $\Xi=\left(\left[\begin{array}{l}G \\ L \\ K\end{array}\right] ;\left[\begin{array}{l}\mathcal{U} \\ \mathcal{X} \\ \mathcal{Y}\end{array}\right]\right)$ be the colligation in Proposition 6.2. Define $\mathcal{Z}^{\prime}$ by $(6.10)$, and let $G^{\prime}=\left.G\right|_{\mathcal{Z}^{\prime}}, L^{\prime}=\left.L\right|_{\mathcal{Z}^{\prime}}, K^{\prime}=\left.K\right|_{\mathcal{Z}^{\prime}}$ and $\mathcal{W}=L^{2}\left(\Gamma_{1}\right)$. Then the colligation $\Xi^{\prime}:=\left(\left[\begin{array}{c}G^{\prime} \\ L^{\prime} \\ K^{\prime}\end{array}\right] ;\left[\begin{array}{c}\mathcal{W} \\ \mathcal{W}\end{array}\right]\right)$ is an impedance conservative boundary node with a unitary semigroup which coincides with the semigroup of $\Xi$.

Proof. The Green-Lagrange identity (3.3) is implied by (3.5) that holds by Proposition 6.2. It is clear from the proof of Proposition 6.2 that $\left[\begin{array}{l}G^{\prime} \\ L^{\prime}\end{array}\right]$ maps $\mathcal{Z}^{\prime}$ onto $\left[\begin{array}{c}\mathcal{W} \\ \mathcal{X}\end{array}\right]$. That $\left[\begin{array}{c}G^{\prime} \\ L^{\prime}\end{array}\right]$ closed (with domain $\mathcal{Z}^{\prime}$ ) follows because $\left[\begin{array}{c}G \\ L\end{array}\right]$ closed (with domain $\mathcal{Z}$ ) and $\mathcal{Z}^{\prime}=\left\{f \in \mathcal{Z}:\left[\begin{array}{c}G \\ L\end{array}\right] f \in\left[\begin{array}{c}\mathcal{W} \\ \mathcal{X}\end{array}\right]\right\}$. We conclude from Theorem 4.3 that $\Xi^{\prime}$ is an impedance conservative internally well-posed boundary node in the sense of Definition 3.2. Since $\mathcal{N}\left(G^{\prime}\right)=\mathcal{N}(G)$, the semigroups of $\Xi^{\prime}$ and $\Xi$ coincide.

Note, in particular, that Proposition 6.3 gives another proof of the scattering conservativity of the example discussed in [MS06a, Section 5] (that node is the Cayley transform of the node in Proposition 6.3 with parameter $\beta=1$ ).

Remark 6.4. If we interchange the input and the output in the node $\Xi^{\prime}$, then the resulting colligation is still impedance conservative, and its main operator $\left.L^{\prime}\right|_{\mathcal{N}(K)^{\prime}}$ is unitary. However, the colligation $\Xi^{\prime}$ is not a boundary node since $K^{\prime}$ is not surjective with values in $L^{2}\left(\Gamma_{1}\right)$.

Let us explain why the colligation $\Xi^{\prime}$ in Proposition 6.3 does not fit into the framework of strong colligations. By Lemma 6.5 below, the space $\mathcal{D o m}\left(\Xi^{\prime}\right)$ is a proper dense subspace $\mathcal{D} o m(\Xi)$. Thus, $\operatorname{Dom}\left(L^{\prime}\right)=\mathcal{D}$ om $\left(\Xi^{\prime}\right)$ is not closed in

\footnotetext{
${ }^{3}$ In fact $x_{0} \in H^{3 / 2}(\Omega)$ by the elliptic regularity theory, but this is not needed here.
} 
$\operatorname{Dom}(L)=\operatorname{Dom}(\Xi)$ (with respect to the graph norm), and hence $L^{\prime}$ is not closed (see Lemma 6.6). We conclude that the colligation $\Xi^{\prime}$ is not strong.

Lemma 6.5. Define $\Xi$ and $\Xi^{\prime}$ as in Propositions 6.2 and 6.3. Then $\mathcal{Z}^{\prime}:=\operatorname{Dom}\left(\Xi^{\prime}\right)$ is a proper dense subspace of $\mathcal{Z}:=\operatorname{Dom}(\Xi)$.

Proof. As we saw in the proof of Proposition 6.2 (in the place where we proved that $\left[\begin{array}{l}G \\ L\end{array}\right]$ is surjective), the operator $\left[\begin{array}{l}G \\ L\end{array}\right]$ is a continuous map of $\mathcal{Z}$ onto $\left[\begin{array}{c}H^{-1 / 2}\left(\Gamma_{2}\right) \\ L^{2}(\Omega)\end{array}\right]$, and this map is both injective and surjective. Thus, it has a bounded inverse $\left[\begin{array}{l}G \\ L\end{array}\right]^{-1}:\left[\begin{array}{c}H^{-1 / 2}\left(\Gamma_{2}\right) \\ L^{2}(\Omega)\end{array}\right] \rightarrow \mathcal{Z}$. By definition $\left[\begin{array}{c}w_{0} \\ u\end{array}\right] \in \mathcal{Z}^{\prime}$ if and only if $\left[\begin{array}{c}w_{0} \\ u\end{array}\right] \in \mathcal{Z}$ and $G w_{0} \in L^{2}\left(\Gamma_{2}\right)$. In other words, $\mathcal{Z}$ is the image of $\left[\begin{array}{c}L^{2}\left(\Gamma_{2}\right) \\ L^{2}(\Omega)\end{array}\right]$ under $\left[\begin{array}{l}G \\ L\end{array}\right]^{-1}$. Since $\left[\begin{array}{l}L^{2}\left(\Gamma_{2}\right) \\ L^{2}(\Omega)\end{array}\right]$ is a proper dense subspace of $\left[\begin{array}{c}H^{-1 / 2}\left(\Gamma_{2}\right) \\ L^{2}(\Omega)\end{array}\right]$, we conclude that $\mathcal{Z}^{\prime}$ is a proper dense subspace of $\mathcal{Z}$.

Lemma 6.6. Let $T$ be a closed operator $\mathcal{X} \rightarrow \mathcal{Y}$, and let $\mathcal{Z}$ be a subspace of $\operatorname{Dom}(T)$. Then $\left.T\right|_{\mathcal{Z}}$ is closed if and only if $\mathcal{Z}$ is a closed subspace of $\mathcal{D} o m(T)$ (with respect to the graph norm of $T$ ).

Proof. Let the sequence $z_{j} \in \mathcal{Z}$ satisfy $z_{j} \rightarrow z$ in $\operatorname{Dom}(T)$, i.e., $z_{j} \rightarrow z$ in $\mathcal{X}$ and $T z_{j}=\left.T\right|_{\mathcal{Z}} z_{j} \rightarrow y$ in $\mathcal{Y}$. On one hand, this implies that $z \in \mathcal{Z}$ if and only if $\left.T\right|_{\mathcal{Z}}$ is closed, and on the other hand, this implies that $z \in \mathcal{Z}$ if and only $\mathcal{Z}$ is a closed subspace of $\mathcal{D} o m(L)$. Thus, $\left.T\right|_{\mathcal{Z}}$ is closed if and only if $\mathcal{Z}$ is closed in $\mathcal{D} o m(T)$.

\section{REFERENCES}

[Bog74] J. Bognár, Indefinite inner product spaces, Ergebnisse der Mathematik und ihrer Grenzgebiete, vol. 78, Springer-Verlag, Berlin, Heidelberg, New York, 1974.

[GG91] V. I. Gorbachuk and M. L. Gorbachuk, Boundary value problems for operator differential equations, Mathematics and its Applications (Soviet Series), vol. 48, Kluwer Academic Publishers Group, Dordrecht, 1991, translation and revised from the 1984 Russian original.

[Gri85] P. Grisvard, Elliptic problems in non-smooth domains, Pitman, Boston, 1985.

[Lag83] J. E. Lagnese, Decay of solutions of wave equations in a bounded region with boundary dissipation, J. Differential Equations 50 (1983), 163-182.

[Lei73] R. Leis, Initial boundary value problems in mathematical physics, John Wiley \& Sons, 1986.

[Liv73] M. S. Livšic, Operators, oscillations, waves (open systems), Translations of Mathematical Monographs, vol. 34, American Mathematical Society, Providence, Rhode Island, 1973.

[LM72] J.-L. Lions and E. Magenes, Non-homogeneous boundary value problems and applications. Vol. I, Springer-Verlag, New York, 1972, translation from the French by P. Kenneth, Die Grundlehren der mathematischen Wissenschaften, Band 181.

[Mal05] J. Malinen, Conservativity and time-flow invertiblity of boundary control systems, Proceedings of CDC-ECC'05, 2005.

[MS06a] J. Malinen and O. J. Staffans, Conservative boundary control systems, J. Differential Equations, to appear.

[MSW06] J. Malinen, O. J. Staffans, and G. Weiss, When is a linear system conservative?, Quart. Appl. Math. 2006.

[Paz83] A. Pazy, Semi-groups of linear operators and applications to partial differential equations, Springer-Verlag, Berlin, 1983.

[Sta02] O. J. Staffans, Passive and conservative continuous-time impedance and scattering systems. Part I: Well-posed systems, Math. Control Signals Systems 15 (2002), 291315. 
[Sta05] O. J. Staffans, Well-posed linear systems, Cambridge University Press, Cambridge, (2005).

Jarmo Malinen, Department of Mathematics, Helsinki University of Technology, P.O.Box 1100, FIN-02015 HUT, Finland. Jarmo.Malinen@hut.fi.

Olof Staffans, Department of Mathematics, Ábo Akademi University, Fin-20500 Áo, Finland, http://www.abo.fi/ ${ }^{\sim}$ staffans. 